Original Article

\title{
Right Axillary Artery Cannulation in Aortic Valve Replacement
}

\author{
Mitsuharu Hosono, MD, Toshihiko Shibata, MD, Takashi Murakami, MD, \\ Masanori Sakaguchi, MD, Yasuo Suehiro, MD, and Shigefumi Suehiro, MD
}

\begin{abstract}
Objective: This retrospective study aimed to evaluate the results of our experience with axillary artery cannulation via a side graft in aortic valve replacement in patients with ascending aortic atherosclerotic disease.

Methods: From January 2002 to 2012, we operated on 76 patients for aortic valve disease with the use of the axillary artery for arterial inflow in our institute. The indications for cannulation of the axillary artery were aortic aneurysm in 37 patients, severe aortic atherosclerosis in 28 patients, and re do surgery in 11 patients.

Results: Right axillary artery cannulation via a side graft provides sufficient antegrade aortic flow of $2.6 \pm 0.1 \mathrm{~L} / \mathrm{m}^{2}$ during cardiopulmonary bypass. No additional arterial cannulation was necessary to obtain sufficient perfusion during cardiopulmonary bypass. Although permanent perioperative stroke was observed in two patients, this did not occur during the operation. There were no problems with cannulation or wound and graft infections. During the follow-up period, there were no thrombotic events due to an axillary graft stump in the right upper extremities.

Conclusions: Axillary artery cannulation via a side graft is a useful and safe option for cardiopulmonary bypass in patients with atherosclerotic disease of the ascending aorta undergoing aortic valve replacement.
\end{abstract}

Keywords: aortic valve replacement, axillary artery cannulation, cardiopulmonary bypass

\section{Introduction}

Atherosclerotic disease in the ascending aorta can complicate the choice of an arterial cannulation site for cardiopulmonary bypass in aortic valve replacement. In some cases of aortic valve replacements, concomitant surgery is necessary (e.g., with aortic aneurysm and coronary artery

Department of Cardiovascular Surgery, Osaka City University Graduate School of Medicine, Osaka, Osaka, Japan

Received: September 28, 2015; Accepted: December 14, 2015 Corresponding author: Mitsuharu Hosono, MD. Department of Cardiovascular Surgery, Osaka City University Graduate School of Medicine, 1-4-3 Asahimachi, Abeno-ku, Osaka, Osaka 545-8585, Japan

Email: mi_hosono@hotmail.com

(C)2016 The Editorial Committee of Annals of Thoracic and Cardiovascular Surgery. All rights reserved. disease). In such cases of atherosclerotic ascending aortic disease, the femoral artery is commonly used as an alternative arterial cannulation site for cardiopulmonary bypass. However, femoral arterial cannulation is associated with a risk of embolization to the brain or the heart with atherosclerotic plaques or thrombi from the thoracic and abdominal aortic walls due to retrograde aortic perfusion. ${ }^{1,2)}$ To avoid the risk of brain or heart embolization, the axillary artery is a good alternative arterial cannulation site. In thoracic aortic surgery and coronary artery bypass grafting, some previous reports have suggested that arterial perfusion using right axillary artery cannulation provides sufficient antegrade aortic flow and less risk of athero-embolic complications. ${ }^{3-5)}$

In our institute, ascending aortic cannulation is the first choice in aortic valve replacement. However, in a patient with severe ascending aortic disease, we use right 
Table 1 Patients' profiles

\begin{tabular}{lc}
\hline Male:Female & $43: 33$ \\
Age & $69.4 \pm 10.1$ \\
Obesity & $11(14.5)$ \\
Hypertension & $43(56.6)$ \\
Hyperlipidemia & $34(44.7)$ \\
Diabetes mellitus & $13(17.1)$ \\
Smoking & $25(32.9)$ \\
Hemodialysis & $11(14.5)$ \\
History of cerebrovascular disease & $8(10.5)$ \\
Liver cirrhosis & $2(2.6)$ \\
\hline
\end{tabular}

Data were expressed as mean \pm SD or direct number (\%). SD: standard deviation

axillary artery cannulation via a side graft as the second alternative cannulation site. We performed a retrospective study to evaluate the results of our experience with axillary artery cannulation via a side graft in aortic valve replacement in patients with ascending aortic atherosclerotic disease.

\section{Patients and Methods}

\section{Patients}

From January 2002 to 2012, we operated on 76 patients for aortic valve disease, using the axillary artery for arterial inflow in our institute. Medical records were retrospectively reviewed for pre-, intra-, and postoperative variables. Preoperative variables are shown in Tables 1 and 2. The mean age of the patients was $69.4 \pm$ 10.1 years old, and 43 were men and 33 were women. The indications for axillary artery cannulation were following, aortic aneurysm in 37 patients, severe aortic atherosclerosis in 28 patients, and re do surgery in 11 patients. In 73 patients, axillary artery cannulation was scheduled. In the other three patients, axillary artery cannulation was decided on during the operation. One of these three patients had an emergency procedure performed because of ascending aortic dissection. Aortic disease was evaluated before the operation by computed tomography and evaluated by epi-aortic ultrasonography during operation.

Solitary aortic valve replacement was performed in 29 patients. Concomitant surgery is shown in Table 3. Conventional cardiopulmonary bypass was used in 45 patients, antegrade selective cerebral perfusion with moderately hypothermic circulatory arrest was used in 30 patients, and circulatory arrest with deep hypothermia was used in one patient.
Table 2 Cardiac disease

\begin{tabular}{lc}
\hline Aortic stenosis & $57(75.0)$ \\
Aortic regurgitation & $12(15.8)$ \\
Prosthetic valve insufficiency & $7(9.2)$ \\
PCI history & $7(9.2)$ \\
Old myocardial infarction & $7(9.2)$ \\
Atrial fibrillation/Atrial flutter & $9(11.8)$ \\
NYHA III, IV & $24(31.6)$ \\
Left ventricular EF <40\% & $6(7.9)$ \\
Coronary artery disease & \\
1 vessel disease & $10(13.2)$ \\
2 vessels disease & $5(6.6)$ \\
3 vessels disease & $3(3.9)$ \\
\hline Data were expressed as direct number $(\%)$. \\
PCI: percutaneous coronary intervention; \\
NYHA: New York Heart Association; \\
EF: ejection fraction
\end{tabular}

Table 3 Operative data

\begin{tabular}{lc} 
Emergency & $3(3.9)$ \\
Re-do operation & $12(15.8)$ \\
Solitary aortic valve replacement & $28(36.8)$ \\
Concomitant operation & \\
$\quad$ Multiple valve & $2(2.6)$ \\
$\quad$ Aortic surgery & $37(48.7)$ \\
$\quad$ Coronary artery bypass grafting & $12(15.8)$ \\
Nicks procedure & $4(5.3)$ \\
Conventional ECC & $45(59.2)$ \\
Antegrade selective cerebral perfusion & $30(39.5)$ \\
Circulatory arrest & $1(1.3)$ \\
Operation time (minutes) & $364.7 \pm 96.2$ \\
ECC time (minutes) & $185.5 \pm 57.1$ \\
Aortic clamping time (minutes) & $126.5 \pm 39.9$ \\
\hline
\end{tabular}

Data were expressed as mean \pm SD or direct number (\%). ECC: extracorporeal circulation; SD: standard deviation; Antegrade selective cerebral perfusion: antegrade selective cerebral perfusion with moderately hypothermic circulatory arrest

\section{Operative technique}

Patients were placed in the standard supine position. Arterial pressure was monitored at the right radial artery and the other peripheral artery. After an infraclavicular skin incision, the pectoralis major fascia was incised and muscle fibres were split. If necessary, the pectoralis pectoris minor muscle was partially or totally divided. The axillary artery was exposed without touching the brachial nerves. After heparinization, an 8-mm polyethylene graft was sewn to the axillary artery using a running 5-0 polypropylene suture in an end-to-side fashion at a $45^{\circ}$ (Fig. 1). All patients were approached via a median sternotomy incision. The 8 -mm graft was then connected to a $22 \mathrm{Fr}$ straight 


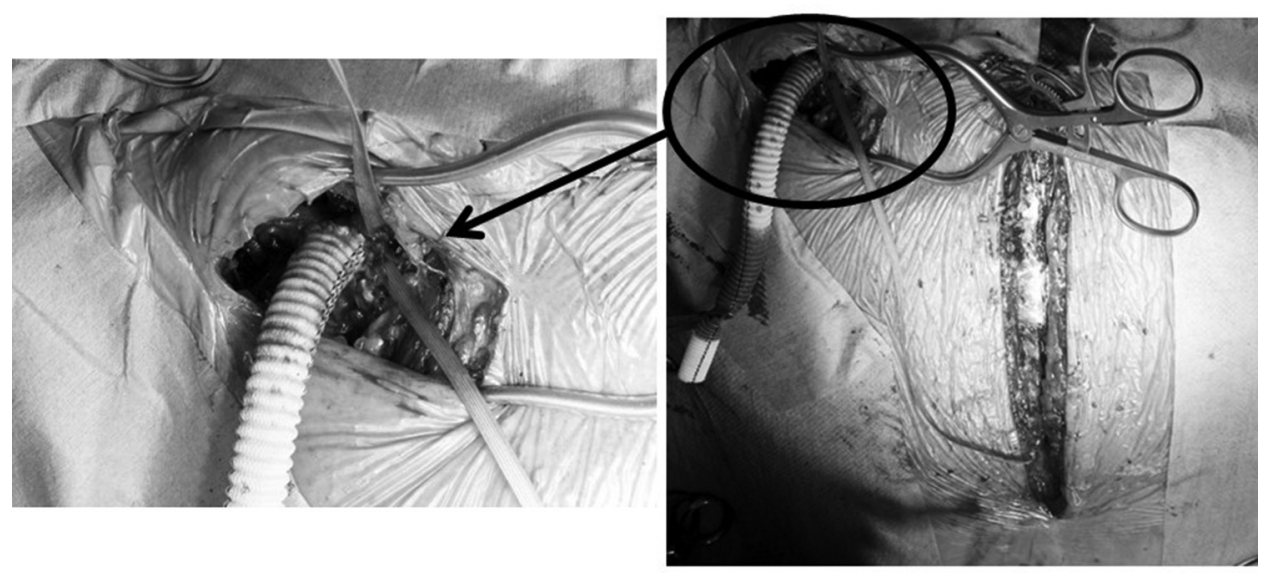

Fig. 1 An 8-mm polyethylene graft was sewn to the right axillary artery using a running 5-0 polypropylene suture in an end-to-side fashion at a $45^{\circ}$.

arterial cannula. A venous cannula was usually placed at the right atrium after sternotomy. Cardiopulmonary bypass was then established. The venting tube was placed in the left ventricle via the right superior pulmonary vein. The ascending aorta was clamped, and myocardial protection was achieved with blood cardioplegic solution in an antegrade and a retrograde fashion. The clamping position was selected by evaluation using epi-aortic ultrasonography.

If selective antegrade cerebral perfusion was necessary, patients were cooled to moderate levels of hypothermia $\left(26^{\circ} \mathrm{C}\right)$. When the rectal temperature reached $26^{\circ} \mathrm{C}$, the cardiopulmonary bypass was discontinued. Selective antegrade cerebral perfusion was achieved by restarting right axillary graft perfusion by clamping the brachiocephalic artery, with direct cannulation to the left carotid artery and to the left axillary artery. Brain perfusion pressure was monitored through the right radial artery.

At the end of the procedure, the axillary artery graft was clamped and cut as short as possible. The graft stump was closed with 5-0 polypropylene running suture.

\section{Data analysis}

All data were obtained by retrospective review of medical records. Written informed consent was obtained from each patient. Statistical analysis was performed with JMP software package (SAS Institute Inc., Cary, NC). Categorical variables are expressed as direct numbers and percentages. Continuous variables are expressed as mean and standard deviation. Operative mortality included in-hospital and 30-day mortality. Permanent perioperative stroke was defined as a new and permanent focal neurological deficit with or without evidence of cerebral infarction on computed tomography or magnetic resonance imaging, and was confirmed by a neurologist.

\section{Results}

\section{During the operation}

There was no severe atherosclerotic disease in the axillary artery in the patients. There were no arterial injuries or technical problems in graft anastomosis. Right axillary artery cannulation via a side graft provided sufficient antegrade aortic flow of $2.6 \pm 0.1 \mathrm{~L} / \mathrm{m}^{2}$ during cardiopulmonary bypass. No additional arterial cannulation was necessary to obtain sufficient perfusion during cardiopulmonary bypass.

\section{Operative results}

There were two hospital deaths (2.6\%). One cause of death was hepatic failure and the other was low output syndrome. Intra-aortic balloon pumping support was necessary in four patients. The mean intensive care unit stay was 1.9 days. The operative results and postoperative complications are shown in Table 4. One patient underwent temporary haemodialysis postoperatively, but no patients required permanent haemodialysis.

Permanent perioperative stroke was observed in two patients. Because one patient had an operation for a thrombosed artificial valve, the time when the cerebral complication occurred was unclear. Stroke in the other patient was occurred 2 days after the operation. None of the patients had cerebrovascular complication during the operation. Furthermore, other embolic events were not observed in any of the patients. 
Table 4 Operative results

$\begin{array}{lc}\text { ICU stay (days) } & 1.9 \pm 1.8 \\ \text { Prolong mechanical ventilation }(>72 \mathrm{~h}) & 3(3.9) \\ \text { Re-exploration for bleeding } & 5(6.6) \\ \text { Low output syndrome } & 3(3.9) \\ \text { Intra-aortic balloon pumping } & 5(6.6) \\ \text { Perioperative myocardial infarction } & 0(0.0) \\ \text { Postoperative hemodialysis } & 1(1.3) \\ \text { Elevation of serum creatine level } & 2(2.6) \\ \text { Deep sternal infection } & 0(0.0) \\ \text { Postoperative Af/AF } & 33(43.4) \\ \text { Hospital death } & 2(2.6)\end{array}$

Data were expressed as mean \pm SD or direct number (\%). ICU: intensive care unit; Af/AF: atrial fibrillation/atrial flutter; SD: standard deviation

\section{Complications of exposure of the axillary artery}

In one patient, medial nerve palsy was observed, but the palsy was resolved 6 months after surgery. Seroma was observed in three patients. In these three patients, the seroma was resolved and vanished after one or two punctures. Re-opening of the subclavian wound for bleeding or other reason was not necessary in any of the patients. None of the patients had subclavian wounds or axillary graft infections. During the follow-up period, there were no thrombotic events due to an axillary graft stump in the right upper extremities.

\section{Discussion}

In aortic valve replacement, the ascending aorta is the standard arterial cannulation site in our hospital. However, in some patients with severe atherosclerosis, the ascending aorta is not suitable for a cannulation site. Embolic events during cardiac operations are serious problems of perioperative morbidity and mortality. ${ }^{1)}$ Cannulation to a severely atherosclerotic ascending aorta can be a cause of stroke. Furthermore, cannulation is difficult in calcified aortic walls. In such patients with a diseased ascending aorta, one of the possible surgical modifications is changing the arterial cannulation site. ${ }^{6-8)}$ Axillary artery cannulation can avoid manipulation of the ascending aorta or aortic arch. Antegrade perfusion via axillary artery cannulation is preferable in patients with aortic atherosclerosis, because femoral arterial cannulation has been reported to be associated with embolic events as a consequence of atherosclerotic thrombi. ${ }^{1,9,10)}$ For these reasons, we adopted axillary artery cannulation in aortic valve surgery in patients with a diseased ascending aorta as a second alternative cannulation site. Furthermore, although the femoral artery is sometimes affected by atherosclerosis, atherosclerosis of the axillary artery is rare. In this study, preoperative evaluation of the axillary artery was not performed in all of the patients, but no atherosclerotic disease was observed in any of the patients. The axillary artery may be used for arterial cannulation in attempted patients with a low risk of embolic events.

In coronary artery bypass grafting with aortic valve replacement, many procedures are added in the ascending aorta. These procedures include proximal anastomosis of the graft, arterial cannulation, aortotomy for valve replacement, placement of antegrade cardioplegic cannula, and aortic cross clamping. Avoiding cannulation of the ascending aorta is helpful for finding areas with no disease between calcification and atherosclerosis in the ascending aortic wall. The calcified ascending aorta is easily diagnosed by palpation or preoperative computed tomography. In our institute, preoperative computed tomography is routinely performed to evaluate the ascending aortic calcification. During the operation, epi-aortic ultrasound scanning is performed to identify the atherosclerotic plaque. The ascending aorta is gently palpated and scanned with careful epi-aortic ultrasound to identify areas with no disease for aortic clamping and a proximal anastomotic site for the saphenous vein or radial artery graft.

In some patients undergoing aortic valve replacement, ascending aortic aneurysm or dilatation is treated concomitantly, and cerebral protection during the operation is necessary in this situation. Use of hypothermic circulatory arrest, retrograde cerebral perfusion, and selective antegrade cerebral perfusion has previously been reported for cerebral protection strategies during thoracic aortic surgery. ${ }^{11,12)}$ In our hospital, we conventionally use selective ante-grade cerebral perfusion with moderately hypothermic circulatory arrest as the first choice. Right axillary arterial cannulation is useful for selective ante-grade cerebral perfusion. ${ }^{13)}$ The majority of strokes during aortic surgery are generally embolic events and are unlikely to be affected by methods of cerebral protection unless the time of circulatory arrest is prolonged. ${ }^{14,15)}$ However, the site of cannulation may play an important role because dislodgement of atheromatous debris with subsequent cerebral embolism may occur with either aortic or femoral cannulation. ${ }^{1,16)}$ Although patients with ascending aortic disease were included in this series, there were no permanent complications associated with selective ante-grade cerebral perfusion via the right axillary artery. Therefore, right axillary artery cannulation is considered to be also useful 
for operations in patients with ascending aortic surgery concomitant with aortic valve replacement.

Axillary artery cannulation is also useful during reoperative heart surgery. ${ }^{17)}$ In re-operative cardiac surgery, median sternotomy can cause catastrophic bleeding because of injury to cardiac structures adherent to the posterior plate of the sternum. In re-operative surgery, severe adhesion is sometimes observed, and dissecting the adhesion around the ascending aorta is difficult. In such cases, axillary or femoral artery cannulation should be performed before sternal opening. In such cases, peripheral arterial cannulation enables to surgeons determine the position of the aortic cross-clamp in a small space in the ascending aorta. However, Crooke et al. ${ }^{18)}$ showed that retrograde arterial perfusion increases the risk of stroke in reoperative mitral valve procedures. Although their study was on the mitral valve procedure, they recommend axillary artery cannulation in redo surgery. Thus, axillary artery cannulation is considered to be suitable more than femoral artery cannulation to decrease the risk of stroke.

A contraindication for the use of the axillary artery for cannulation is known severe atherosclerotic disease of the axillary or subclavian artery. However, these vessels are rarely involved in atherosclerotic disease. ${ }^{19)}$ In our study, there was no case of conversion to the other cannulation site. A previous report showed conversion to single femoral artery cannulation because of problems with cannulation in patients with direct axillary artery cannulation. ${ }^{20)} \mathrm{In}$ this study, all cannulations to the axillary arteries were performed through a side graft anastomosed to the axillary artery. Right axillary artery cannulation via a side graft has been reported to have a lower risk of cannulation related morbidity and stroke compared with direct cannulation. ${ }^{16,21)}$ Some complications and pitfalls related to the right arterial cannulation have been reported including arterial injury and dissection, malperfusion and insufficient flow with high resistance. ${ }^{22,23)}$ However, in our series, there was no arterial complication or insufficient flow. We believe that using a side graft is a preferable cannulation technique for the right axillary artery.

Axillary artery cannulation via a side graft allows for safe arterial access avoiding aortic manipulation in aortic valve replacement. This is also useful for establishing antegrade cerebral perfusion during concomitant aortic surgery. Furthermore, avoiding cannulation of the ascending aorta is helpful to find no-diseased areas between calcification and atherosclerosis in the ascending aortic wall. In summary, the axillary artery cannulation is a useful and safe technique for cardiopulmonary bypass in patients with severe atherosclerotic or aneurysmal disease of the ascending aorta undergoing aortic valve replacement.

\section{Disclosure Statement}

None of authors had a conflict of interest concerning this study, and none received outside support for this research.

\section{References}

1) Sabik JF, Lytle BW, McCarthy PM, et al. Axillary artery: an alternative site of arterial cannulation for patients with extensive aortic and peripheral vascular disease. J Thorac Cardiovasc Surg 1995; 109: 885-90; discussion 890-1.

2) Bichell DP, Balaguer JM, Aranki SF, et al. Axilloaxillary cardiopulmonary bypass: a practical alternative to femorofemoral bypass. Ann Thorac Surg 1997; 64: 702-5.

3) Halkos ME, Kerendi F, Myung R, et al. Selective antegrade cerebral perfusion via right axillary artery cannulation reduces morbidity and mortality after proximal aortic surgery. J Thorac Cardiovasc Surg 2009; 138: 1081-9.

4) Kokotsakis J, Lazopoulos G, Milonakis M, et al. Right axillary artery cannulation for surgical management of the hostile ascending aorta. Tex Heart Inst J 2005; 32: 189-93; discussion 185.

5) Leyh RG, Bartels C, Nötzold A, et al. Management of porcelain aorta during coronary artery bypass grafting. Ann Thorac Surg 1999; 67: 986-8.

6) Mills NL, Everson CT. Atherosclerosis of the ascending aorta and coronary artery bypass. Pathology, clinical correlates, and operative management. J Thorac Cardiovasc Surg 1991; 102: 546-53.

7) Wareing TH, Davila-Roman VG, Barzilai B, et al. Management of the severely atherosclerotic ascending aorta during cardiac operations. A strategy for detection and treatment. J Thorac Cardiovasc Surg 1992; 103: 453-62.

8) Culliford AT, Colvin SB, Rohrer K, et al. The atherosclerotic ascending aorta and transverse arch: a new technique to prevent cerebral injury during bypass: experience with 13 patients. Ann Thorac Surg 1986; 41: 27-35.

9) Martin WR, Hashimoto SA. Stroke in coronary bypass surgery. Can J Neurol Sci 1982; 9: 21-6.

10) Price DL, Harris J. Cholesterol emboli in cerebral arteries as a complication of retrograde aortic perfusion during cardiac surgery. Neurology 1970; 20: 1209-14.

11) Niwa $H$, Nara $M$, Kimura $T$, et al. Prolongation of total permissible circulatory arrest duration by deep hypothermic intermittent circulatory arrest. J Thorac Cardiovasc Surg 1998; 116: 163-70.

12) Higami $T$, Kozawa $S$, Asada $T$, et al. Retrograde cerebral perfusion versus selective cerebral perfusion as evaluated 
by cerebral oxygen saturation during aortic arch reconstruction. Ann Thorac Surg 1999; 67: 1091-6.

13) Budde JM, Serna DL Jr., Osborne SC, et al. Axillary cannulation for proximal aortic surgery is as safe in the emergent setting as in elective cases. Ann Thorac Surg 2006; 82: 2154-9; discussion 2159-60.

14) Di Eusanio M, Schepens MAAM, Morshuis WJ, et al. Antegrade selective cerebral perfusion during operations on the thoracic aorta: factors influencing survival and neurologic outcome in 413 patients. J Thorac Cardiovasc Surg 2002; 124: 1080-6.

15) Kazui T, Yamashita K, Washiayama N, et al. Usefulness of antegrade selective cerebral perfusion during aortic arch operations. Ann Thorac Surg 2002; 74: S1806-9; discussion S1825-32.

16) Svensson LG, Blackstone EH, Rajeswaran J, et al. Does the arterial cannulation site for circulatory arrest influence stroke risk? Ann Thorac Surg 2004; 78: 127484; discussion 1283-84.

17) Mastroroberto P, Chello M, Di Virgilio A, et al. Usefulness of the axillary artery as vascular access for cardiopulmonary bypass. J Cardiovasc Surg (Torino) 2006; 47: 457-60.
18) Crooke GA, Schwartz CF, Ribakove GH, et al. Retrograde arterial perfusion, not incision location, significantly increases the risk of stroke in reoperative mitral valve procedures. Ann Thorac Surg 2010; 89: 723-29; discussion 729-30.

19) Pasic M, Schubel J, Bauer M, et al. Cannulation of the right axillary artery for surgery of acute type A aortic dissection. Eur J Cardiothorac Surg 2003; 24: 231-5; discussion 235-6.

20) Hiraoka A, Chikazawa G, Totsugawa T, et al. Efficacy of right axillary artery perfusion for antegrade cerebral perfusion in open total arch repair. J Vasc Surg 2014; 60: $436-42$.

21) Sabik JF, Nemeh H, Lytle BW, et al. Cannulation of the axillary artery with a side graft reduces morbidity. Ann Thorac Surg 2004; 77: 1315-20.

22) Sinclair MC, Singer RL, Manley NJ, et al. Cannulation of the axillary artery for cardiopulmonary bypass: safeguards and pitfalls. Ann Thorac Surg 2003; 75: 931-4.

23) Schachner T, Nagiller J, Zimmer A, et al. Technical problems and complications of axillary artery cannulation. Eur J Cardiothorac Surg 2005; 27: 634-7. 